\title{
Complete controllability of finite quantum systems with two-fold energy level degeneracy
}

\author{
Zhedong Zhang and H. C. Fu $⿴$ \\ School of Physical Sciences and Technology, Shenzhen University, Shenzhen \\ 518060, P. R. China
}

\begin{abstract}
Complete controllability of finite dimensional quantum systems with energy level degeneracy is investigated using two different approaches. One approach is to apply a weak constant field to eliminate the degeneracy and then control it using techniques developed for non-degenerate quantum systems. Conditions for the elimination of degeneracy are found and the issue of influence of relaxation time of constant external field to the target state are addressed through the fidelity. Another approach is to control the degenerate system by a single control field directly. It is found that the system with two-fold degenerate excited states and non-degenerate ground state are completely controllable except for the two-level system. Conditions of complete controllability are found for both systems with different energy gaps and with equal energy gaps.
\end{abstract}

$\ddagger$ E-mail: hcfu@szu.edu.cn 
Complete controllability of finite quantum systems with two-fold energy level degeneracy2

\section{Introduction}

Quantum control is essentially understood as a coherent or incoherent manipulation of a quantum system, which attempts a time evolution from an arbitrary initial state to an arbitrarily given target state 1, 2, 3, 4. It was first proposed by Huang et. al. in 1983 [5] and was then further developed for application in the control of chemical reactions. In recent years, the controllability of quantum system has been well defined [6, 7, 8] and investigated using algebraic methods [9, 10, graph methods [11, 12] and transfer graph methods [13. Cabrera et. al. investigated a sufficient condition for the state control of symmetric degenerate quantum systems and found that the underlying Lie algebra could be the symplectic algebra rather than $\operatorname{su}(N)$ 14]. However, it seems that the control of quantum systems with energy level degeneracy has not been investigated systematically in the literature and it is obviously significant to investigate quantum control of systems with energy level degeneracy.

In this paper, we shall present two different approaches to the controllability of finite dimensional quantum system with two-fold degenerate excited states and non-degenerate ground state. One approach is to first eliminate the degeneracy using a constant excitation field and then control it using the techniques developed for the control of non-degenerate system. In this approach one has to choose the proper external excitation field such that the energy structure of the degeneracyeliminated system meets the controllability criteria for the complete controllability of non-degenerate system [9, 10. In quantum control we generally require that the control fields attenuate to zero when the target state is achieved. However, the excitation field is a constant field and we cannot switch it off instantly when the target state is achieved, rather the excitation field tends to zero in a period of time (relaxation time) and this will definitely cause a shift of target state. We shall examine how much the final state shifted from the target state by evaluating their fidelity. We find that the shift of final state from the target state is minor if the decay time is short enough.

Another approach is to use a single external control field to control the degenerate system directly without eliminating energy degeneracy first. Complete controllability is systematically investigated using Lie algebra method. Lie algebra method is an important method for the investigation of both classical [15] control theory and quantum control theory [9, 10, 16. It is found that the degenerate systems can be completely controlled through a single control field if some conditions are fulfilled except the simplest two energy level system. Controllability conditions are algebraically found.

This paper is organized as follows. In Sec. II we formulate two control schemes and fix some notations. In Sec. III we investigate the control of degenerate system by eliminating the degeneracy using constant excitation field. In Sec. IV we turn to the direct control of degenerate system using single control field. We conclude in Sec. V.

\section{Control of the system with energy degeneracy}

Consider an $N$-level quantum system described by the following Hamiltonian

$$
H_{0}=\sum_{n=1}^{N} \sum_{k=1}^{\beta_{n}} E_{n}|n, k\rangle\langle n, k|,
$$


Complete controllability of finite quantum systems with two-fold energy level degeneracy3

where $E_{n}$ is the eigen energy of the $n$-th level and $|n, k\rangle$ are corresponding eigenstates. Here we only consider the case where the ground state is non-degenerate and all excited states are two-fold degenerate, namely

$$
\beta_{n}= \begin{cases}1, & \text { when } n=1 \\ 2, & \text { when } n \geq 2 .\end{cases}
$$

Our aim is to steer the system (11) to an arbitrary given target state by interacting with classical fields. As the controllability of finite systems without degeneracy has been extensively studied [6, 9, 10, a natural idea is to apply a constant excitation field to eliminate the energy degeneracy and then control it using the techniques developed for the non-degenerate system. Suppose that the interaction Hamiltonian $H_{\mathrm{e}}$ between the excitation field and the system takes the following form

$$
\begin{aligned}
H_{\mathrm{e}}= & \sum_{n=1}^{N-1} \sum_{k=1}^{\beta_{n}} \sum_{p=1}^{2} g_{n k, n+1 p}(|n, k\rangle\langle n+1, p|+| n+1, p\rangle\langle n, k|) \\
& +\sum_{n=2}^{N} g_{n 1, n 2}(|n, 1\rangle\langle n, 2|+| n, 2\rangle\langle n, 1|)
\end{aligned}
$$

where $g_{m n, p q}$ are real constants. Then apply the control fields to control the excited system. In this paper, we are particularly interested in the case of a single control field. In this case the interaction Hamiltonian takes the following dipole form

$$
H_{I}=\sum_{n=1}^{N-1} \sum_{k=1}^{\beta_{n}} \sum_{p=1}^{2} d_{n k, n+1 p}(|n, k\rangle\langle n+1, p|+| n+1, p\rangle\langle n, k|) .
$$

Then the total Hamiltonian of the control system is

$$
H=H_{0}+H_{\mathrm{e}}+f(t) H_{I},
$$

where $f(t)$ is the classical control field. For this scheme we need to address two issues:

(1) After the degeneracy is eliminated, whether the degeneracy-eliminated system meets the controllability criteria given in the [9, 10];

(2) In quantum control, we generally require that the control fields approach to 0 when the control time $T$ is reached and the target state is achieved. But for the constant excitation field removing the degeneracy, we have to turn it off when the target state is archived and this relaxation needs time which may cause the target state a shift. So we need to answer how the relaxation time affect the target state.

We will address both issues in Sec. 3 .

Another approach is to control the system (11) using the control field (4) directly, just like the control of non-degenerate system. In this case the Hamiltonian of the total control system is

$$
H=H_{0}+f(t) H_{I} .
$$

We will see in Sec. 4 that the system can be completely controlled if some conditions about the coupling constants in $H_{I}$ are satisfied except the simplest two energy level system. 
Let us fix some notations we will use hereafter. For convenience, let us denote $e_{i j, k l}=|i, j\rangle\langle k, l|$ and define the following skew-Hermitian operators

$$
\begin{aligned}
& x_{n k, m l}=i(|n, k\rangle\langle m, l|+| m, l\rangle\langle n, k|), \quad n<m, \\
& y_{n k, m l}=|n, k\rangle\langle m, l|-| m, l\rangle\langle n, k|, \quad n<m, \\
& h_{n k, m l}=i(|n, k\rangle\langle n, k|-| m, l\rangle\langle m, l|), \quad n<m,
\end{aligned}
$$

on the Hilbert space of the considered system with dimension $2(N-1)+1=2 N-1$. Those operators generate the Lie algebra $\operatorname{su}(2 N-1)$. To prove the complete controllability, we need to prove that the Lie algebra $L_{0}$ generated by $i H_{0}$ and $i H_{I}$ is $\mathrm{su}(2 N-1)$, or in other words, generate all operators in (7). In fact, we only need to prove that $i H_{0}$ and $i H_{I}$ generate the following operators

$$
x_{n k, n+1 l}, \quad y_{n k, n+1 l}, \quad h_{n k, n+1 l}, \quad 1 \leq n \leq N-1 ; k, l=1,2,
$$

as from those operators we further have

$$
\begin{aligned}
& {\left[x_{n k, n+1 l}, y_{n+1 l, n+2 p}\right]=x_{n k, n+2},} \\
& {\left[y_{n k, n+1 l}, y_{n+1 l, n+2 p}\right]=y_{n k, n+2 p},} \\
& -2^{-1}\left[x_{n k, n+2 p}, y_{n k, n+2 p}\right]=h_{n k, n+2 p},
\end{aligned}
$$

and then all elements in (7) recurrently. Therefore the system is completely controllable if elements (8) can be generated by $i H_{0}$ and $i H_{I}$.

\section{Control by elimination of degeneracy}

Let us first briefly discuss conditions of complete controllability of degenerate systems by eliminating degeneracy through a constant classical field. Suppose that the excitation field is week enough that we can use the perturbation theory to evaluate the new energy structure of the system. It is easy to find the first order approximation of the eigen energy

$$
E_{n k}=E_{n}+E_{n k}^{(1)},
$$

where $E_{n 1}^{(1)}=-\left|g_{n 1, n 2}\right|$ and $E_{n 2}^{(1)}=\left|g_{n 1, n 2}\right|$. As the excitation field is week, we can require the degeneracy-removed system does not have energy level crossing

$$
E_{n+1,1}>E_{n, 2} .
$$

To meet the controllability criteria for non-degenerate system given in [9, 10, we require that, for example, the first energy gap $\left(E_{2}-\Gamma_{2}\right)-E_{1}$ is different from any others, namely

$$
\begin{gathered}
\left(E_{2}-\Gamma_{2}\right)-E_{1} \neq\left(E_{n}+\Gamma_{n}\right)-\left(E_{n}-\Gamma_{n}\right)=2 \Gamma_{n}, \\
\left(E_{2}-\Gamma_{2}\right)-E_{1} \neq\left(E_{n}-\Gamma_{n}\right)-\left(E_{n-1}+\Gamma_{n-1}\right), \\
(n=2,3, \ldots, 2 N-1),
\end{gathered}
$$

by proper choice of the coupling constants $g_{n 1, n 2}$. The system is thus completely controllable if conditions (11) and (12) are fulfilled.

We then address the issue of the influence of relaxation time to the target state. Assume that the system has been driven to the normalized target state at time $T$

$$
|\psi(T)\rangle=\sum_{n=1}^{N} \sum_{p=1}^{\beta_{n}} C_{n p}(T)|n, p\rangle .
$$


Complete controllability of finite quantum systems with two-fold energy level degeneracy5

We then switch off the constant excitation field and, without losing generality, we assume that this process is governed by the Hamiltonian

$$
H=H_{0}+e^{-(t-T) / \tau} H_{e}, \quad t \geq T,
$$

where $\tau$ is the relaxation time. From standard time-dependent perturbation theory, we can easily obtain the the state of the first order at the time of half decay $T_{e}=\tau \ln 2+T$

$$
\left|\psi_{I}\left(T_{e}\right)\right\rangle=\sum_{m=1}^{N} \sum_{k=1}^{\beta_{m}}\left(C_{m k}(T)+C_{m k}^{(1)}\left(T_{e}\right)\right)|m, k\rangle,
$$

where

$$
\begin{aligned}
C_{m k}^{(1)}\left(T_{e}\right)= & \sum_{p=1}^{\beta_{m-1}} E_{m, m-1}(\tau)\left(1-\frac{1}{2} e^{i \omega_{m, m-1} \tau \ln 2}\right) C_{m-1, k}(T) g_{m k, m-1 p} \\
& +\sum_{p=1}^{\beta_{n}} \frac{\tau}{2 i \hbar} C_{m p}(T) g_{m k, m p} \\
& +\sum_{p=1}^{\beta_{m+1}} E_{m, m+1}(\tau)\left(1-\frac{1}{2} e^{i \omega_{m, m+1} \tau \ln 2}\right) C_{m+1, k}(T) g_{m k, m+1 p}
\end{aligned}
$$

and $\omega_{m n}=\left(E_{m}-E_{n}\right) / \hbar, g_{m k, n p}=\left\langle m, k\left|H_{e}\right| n, p\right\rangle, E_{m, m \pm 1}(\tau)=1 /\left(E_{m}-E_{m \pm 1}+\right.$ $i \hbar / \tau)$.

To compare the difference between target state and $\left|\psi_{I}\left(T_{e}\right)\right\rangle$, we examine the fidelity $F$ between two states

$$
F \equiv \frac{\left|\left\langle\psi(T) \mid \psi_{I}\left(T_{e}\right)\right\rangle\right|^{2}}{\langle\psi(T) \mid \psi(T)\rangle\left\langle\psi_{I}\left(T_{e}\right) \mid \psi_{I}\left(T_{e}\right)\right\rangle}=\frac{\left|\left\langle\psi(T) \mid \psi_{I}\left(T_{e}\right)\right\rangle\right|^{2}}{\left\langle\psi_{I}\left(T_{e}\right) \mid \psi_{I}\left(T_{e}\right)\right\rangle} .
$$

It is obvious that in the ideal case $\tau \rightarrow 0, C_{m k}^{(1)}\left(T_{e}\right) \rightarrow 0$ and thus $F \rightarrow 1$, as we expected. As the excitation field is week, one generally has $\left|H_{m k, n p} /\left(E_{m}-E_{n}\right)\right| \ll 1$. So if the relaxation time $\tau$ is short enough (This means generally it is much less than the characteristic time of the atom, $\left.\sim 10^{-11} \mathrm{~s}\right), C_{m k}^{(1)}$ is small and the fidelity between the target state and final state is close to 1 .

In Fig 1 we plot the fidelity against the relaxation time $\tau$ for two level system with ground state non-degenerate and excited state two-fold degenerate. It is easy to see that the fidelity depends on the ratio of coupling density and energy gap sensitively. In fact, the perturbation theory requires that the density matrix elements are much smaller than the energy gap. If the ratio is $10^{-3}$ or $10^{-4}$, the fidelity is quite close to 1 and shift of final state from the target state is minor. For ratio is about $10^{-3}$, we find that the fidelity is getting smaller to $95 \%$ when the relaxation time is $10^{-12} \mathrm{~s}$.

\section{Control without system excitation}

In previous section, we presented a scheme of control of degenerate system through eliminating degeneracy by constant excitation field. We naturally ask whether we can control the degenerate systems directly without removing the degeneracy. In this section, we shall address this issue.

Define the energy gap $\mu_{i}=E_{i+1}-E_{i}>0(i=1,2, \cdots, N-1)$ hereafter.

We start by proving the following lemma. 
Complete controllability of finite quantum systems with two-fold energy level degeneracy6

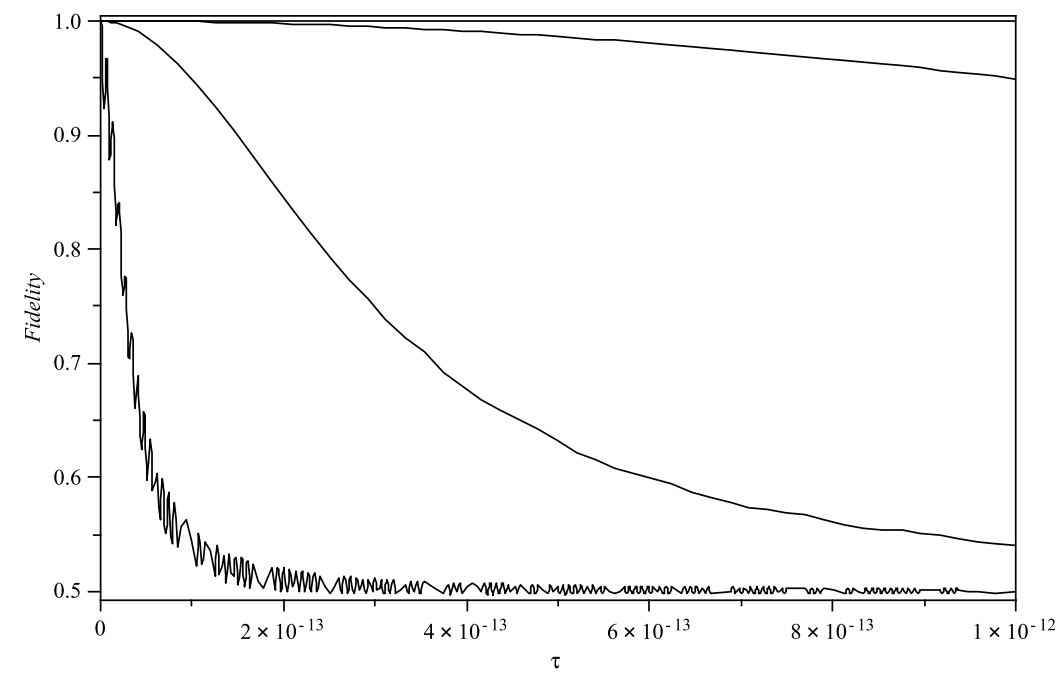

Figure 1. Fidelity against relaxation time $\tau$. The energy gap $E_{2}-E_{1}$ is chosen as $1 \mathrm{eV}$ and $C_{11}, C_{21}, C_{22}$ are chosen as $1 / \sqrt{2}, 1 / 2,1 / 2$ respectively. Corresponding to four curves from top to bottom, we take matrix elements $g_{11,21}=g_{11,22}$ which are chosen as $10^{-23}, 10^{-22}, 10^{-21}$ and $10^{-20}$, respectively.

Lemma 1 If the condition

$$
d_{n 1, n+11} d_{n 2, n+12} \neq d_{n 1, n+12} d_{n 2, n+11}, \quad(2 \leq n \leq N-1),
$$

is satisfied, and $x_{11,21}, x_{11,22} \in L_{0}$ or $y_{11,21}, y_{11,22} \in L_{0}$, then $L_{0}=\operatorname{su}(2 N-1)$ when $N \geq 3$ and the system is completely controllable.

Proof. From $x_{11,21}, x_{11,22} \in L_{0}$, we obtain

$$
\begin{aligned}
& \mu_{1}^{-1}\left[i H_{0}, x_{11,21}\right]=y_{11,21} \in L_{0}, \\
& \mu_{1}^{-1}\left[i H_{0}, x_{11,22}\right]=y_{11,22} \in L_{0}, \\
& -2^{-1}\left[x_{11,21}, y_{11,21}\right]=h_{11,21} \in L_{0}, \\
& -2^{-1}\left[x_{11,22}, y_{11,22}\right]=h_{11,22} \in L_{0},
\end{aligned}
$$

and

$$
\begin{aligned}
V_{0} & =i H_{0}-\left(d_{11,21} x_{11,21}+d_{11,22} x_{11,22}\right) \\
& =\sum_{n=2}^{N-1} \sum_{p=1}^{2} \sum_{k=1}^{2} d_{n p, n+1 k} x_{n p, n+1 k} \in L_{0} .
\end{aligned}
$$

Then we have

$$
\begin{gathered}
{\left[h_{11,21}, V_{0}\right]=d_{21,31} y_{21,31}+d_{21,32} y_{21,32} \in L_{0},} \\
{\left[h_{11,22}, V_{0}\right]=d_{22,31} y_{22,31}+d_{22,32} y_{22,32} \in L_{0},} \\
-\mu_{2}^{-1}\left[i H_{0}, d_{21,31} y_{21,31}+d_{21,32} y_{21,32}\right] \\
=d_{21,31} x_{21,31}+d_{21,32} x_{21,32} \in L_{0}, \\
-\mu_{2}^{-1}\left[i H_{0}, d_{22,31} y_{22,31}+d_{22,32} y_{22,32}\right] \\
=d_{22,31} x_{22,31}+d_{22,32} x_{22,32} \in L_{0} .
\end{gathered}
$$


Complete controllability of finite quantum systems with two-fold energy level degeneracy 7

On the other hand, we also have

$$
\begin{aligned}
& -\left[x_{11,21}, x_{11,22}\right]=y_{21,22}, \\
& -\left[y_{21,22}, d_{21,31} x_{21,31}+d_{21,32} x_{21,32}\right]=d_{21,31} x_{22,31}+d_{21,32} x_{22,32}, \\
& {\left[y_{21,22}, d_{22,31} x_{22,31}+d_{22,32} x_{22,32}\right]=d_{22,31} x_{21,31}+d_{22,32} x_{21,32} .}
\end{aligned}
$$

From Eqs.(21,24), under the condition $d_{21,32} d_{22,31} \neq d_{21,31} d_{22,32}$, we find that $x_{21,31}, x_{21,32} \in L_{0}$, and similarly, from Eqs.(22), 231), we have $x_{22,31}, x_{22,32} \in L_{0}$. Repeating this process for $N-2$ times, we can finally have $x_{n p, n+1 k} \in L_{0}(1 \leq$ $n \leq N-1 ; p, k=1,2)$, and $y_{n p, n+1 k} \in L_{0}(1 \leq n \leq N-1 ; p, k=1,2)$ by evaluating their commutation relation with $i H_{0}$. Those operators generate the Lie algebra $L_{0}=\operatorname{su}(2 N-1)$.

\subsection{The controllability of two-level system}

Let us first consider the simplest case, the two-level system with non-degenerate ground state and two-fold degenerate excited state. The interaction Hamiltonian of the system and control field can be written as

$$
i H_{I}=d_{1} x_{11,21}+d_{2} x_{11,22},
$$

where we have written $d_{1} \equiv d_{11,21}$ and $d_{2} \equiv d_{11,22}$ for convenience. The dynamical Lie algebra $L_{0}$ is generated by $i H_{0}$ and $i H_{1}$. It is easy to see that

$$
\begin{aligned}
& {\left[i H_{0}, i H_{I}\right]=\mu i H_{I}^{1},} \\
& i H_{I}^{1}=d_{1} y_{11,21}+d_{2} y_{11,22} \in L_{0},
\end{aligned}
$$

where $\mu=E_{2}-E_{1}$. Commutation relations

$$
\begin{aligned}
& {\left[i H_{I}, i H_{I}^{1}\right]=2 i H_{I}^{2} \in L_{0},} \\
& i H_{I}^{2}=d_{1}^{2} h_{21,11}+d_{1} d_{2} x_{21,22}+d_{2}^{2} h_{22,11},
\end{aligned}
$$

give rise to a new Lie algebra element $i H_{I}^{2}$. Four elements $\left\{i H_{0}, i H_{I}, i H_{I}^{1}, i H_{I}^{2}\right\}$ span an 4-dimensional Lie algebra with the following closed Lie product

$$
\begin{aligned}
& {\left[i H_{0}, i H_{I}\right]=\mu i H_{I}^{1}, \quad\left[i H_{0}, i H_{I}^{1}\right]=-\mu i H_{I}, \quad\left[i H_{0}, i H_{I}^{2}\right]=0,} \\
& {\left[i H_{I}, i H_{I}^{2}\right]=-2\left(d_{1}^{2}+d_{2}^{2}\right) i H_{I}^{1}, \quad\left[i H_{I}^{1}, i H_{I}^{2}\right]=2\left(d_{1}^{2}+d_{2}^{2}\right) i H_{I} .}
\end{aligned}
$$

From (28) and (31), we find that $\left\{i H_{I}, i H_{I}^{1}, i H_{I}^{2}\right\}$ span an 3-dimensional Lie algebra. Define

$$
i x=\frac{1}{\sqrt{2\left(d_{1}^{2}+d_{2}^{2}\right)}} i H_{I}, \quad i y=\frac{1}{\sqrt{2\left(d_{1}^{2}+d_{2}^{2}\right)}} i H_{I}^{1}, \quad i z=\frac{1}{\sqrt{2\left(d_{1}^{2}+d_{2}^{2}\right)}} i H_{I}^{2},
$$

we find the standard commutation relations of $\mathrm{su}(2)$

$$
[i x, i y]=i z, \quad[i y, i z]=i x, \quad[i z, i x]=i y .
$$

Defining a new element $i h_{0}$ to replace $i H_{0}$

$$
i h_{0} \equiv i H_{0}-\frac{\mu}{2\left(d_{1}^{2}+d_{2}^{2}\right)} i H_{I}^{2},
$$

we can check that

$$
\left[i h_{0}, i x\right]=\left[i h_{0}, i y\right]=\left[i h_{0}, i z\right]=0 .
$$

Therefore, for 2-level degenerate system, the Lie algebra generated by $i H_{0}$ and $i H_{I}$ is $\mathrm{su}(2) \oplus \mathrm{u}(1)$, not $\mathrm{su}(3)$, namely, the system is not completely controllable. 
Complete controllability of finite quantum systems with two-fold energy level degeneracy 8

\subsection{Three level system}

For explicitness, we consider the three-level system in this subsection and we will see that this system is completely controllable if some conditions are satisfied. The system Hamiltonian and the interaction Hamiltonian can be explicitly written as

$$
\begin{aligned}
H_{0}= & E_{1} e_{11,11}+E_{2} e_{21,21}+E_{2} e_{22,22}+E_{3} e_{31,31}+E_{3} e_{32,32}, \\
i H_{1}= & d_{11,21} x_{11,21}+d_{11,22} x_{11,22}+d_{21,31} x_{21,31}+d_{21,32} x_{21,32} \\
& +d_{22,31} x_{22,31}+d_{22,32} x_{22,32} .
\end{aligned}
$$

It is easy to find that

$$
\begin{aligned}
\tilde{V}_{1} & =-\left[i H_{0},\left[i H_{0}, i H_{1}\right]\right]-\mu_{2}^{2}\left(i H_{1}\right) \\
& =\left(\mu_{1}^{2}-\mu_{2}^{2}\right)\left(d_{11,21} x_{11,21}+d_{11,22} x_{11,22}\right) \in L_{0} .
\end{aligned}
$$

Let us consider two different cases.

Case I: $\mu_{1}^{2} \neq \mu_{2}^{2}$. In this case, from (37), we can obtain that

$$
\begin{aligned}
& V_{1}^{\prime}=d_{11,21} x_{11,21}+d_{11,22} x_{11,22} \in L_{0}, \\
& V_{1}=\mu_{1}^{-1}\left[i H_{0}, V_{1}^{\prime}\right]=d_{11,21} y_{11,21}+d_{11,22} y_{11,22} \in L_{0} .
\end{aligned}
$$

It should be noted that $V_{1}, V_{1}^{\prime}$ and

$$
\begin{aligned}
V_{0}= & \frac{1}{2}\left[V_{1}, V_{1}^{\prime}\right]=i\left(d_{11,21}^{2}+d_{11,22}^{2}\right) e_{11,11}-i d_{11,21}^{2} e_{21,21} \\
& -i d_{11,22}^{2} e_{22,22}-d_{11,21} d_{11,22} x_{21,22},
\end{aligned}
$$

generate the Lie algebra $\mathrm{su}(2)$. For the two-level system discussed in previous subsection, except $i H_{0}$, we cannot generate any other elements as we cannot separate the sum (38|39) into $x_{11,21} \in L_{0}$ and $x_{11,22} \in L_{0}$. But fortunately, for $N$-level system $(N \geq 3)$, this can be achieved with the help of terms $x_{2 i, 3 j}$ in $i H_{1}$. To see this, we can evaluate the commutator of $V_{1}$ with $i H_{1}-V_{1}^{\prime}$

$$
\begin{aligned}
V_{2}= & {\left[V_{1}, i H_{1}-V_{1}^{\prime}\right]=\left(d_{11,21} d_{21,31}+d_{11,22} d_{22,31}\right) y_{11,31} } \\
& +\left(\left(d_{11,21} d_{21,32}+d_{11,22} d_{22,32}\right) y_{11,32},\right.
\end{aligned}
$$

and

$$
\begin{aligned}
V_{3} & =\mu_{1}^{-1}\left[i H_{0},\left[V_{2}, i H_{1}-V_{1}^{\prime}\right]\right] \\
& =\left(p d_{21,31}+q d_{21,32}\right) y_{11,21}+\left(p d_{22,31}+q d_{22,32}\right) y_{11,22},
\end{aligned}
$$

where

$$
p=d_{11,21} d_{21,31}+d_{11,22} d_{22,31}, \quad q=d_{11,21} d_{21,32}+d_{11,22} d_{22,32} .
$$

From (39) and (42), we obtain $y_{11,21}, y_{11,22} \in L_{0}$, when

$$
d_{11,21}\left(p d_{22,31}+q d_{22,32}\right) \neq d_{11,22}\left(p d_{21,31}+q d_{21,32}\right) \text {. }
$$

If we further require $d_{21,31} d_{22,32} \neq d_{21,32} d_{22,31}$, from Lemma 1, we conclude that the 3 -level system with different energy gaps is completely controllable.

Case II: $\mu_{1}=\mu_{2}=\mu$. 
Complete controllability of finite quantum systems with two-fold energy level degeneracy9

In this case, Eq. (37) is vanishing and no new element is generated. We can verify that

$$
\begin{aligned}
V_{1} \equiv \mu^{-1}[ & \left.i H_{0}, i H_{1}\right]=d_{11,21} y_{11,21}+d_{11,22} y_{11,22}+d_{21,31} y_{21,31} \\
& +d_{21,32} y_{21,32}+d_{22,31} y_{22,31}+d_{22,32} y_{22,32} \in L_{0} \\
V_{0}=\frac{1}{2}\left[V_{1},\right. & \left.i H_{1}\right]=i\left(d_{11,21}^{2}+d_{11,22}^{2}\right) e_{11,11}+i\left(d_{21,31}^{2}+d_{21,32}^{2}-d_{11,21}^{2}\right) e_{21,21} \\
& +i\left(d_{22,31}^{2}+d_{22,32}^{2}-d_{11,21}^{2}\right) e_{22,22}-i\left(d_{21,31}^{2}+d_{22,31}^{2}\right) e_{31,31} \\
& -i\left(d_{21,32}^{2}+d_{22,32}^{2}\right) e_{32,32} \\
& -\left(d_{11,21} d_{11,22}-d_{21,31} d_{22,31}-d_{21,32} d_{22,32}\right) x_{21,22} \\
& -\left(d_{21,31} d_{21,32}+d_{22,31} d_{22,32}\right) x_{31,32} \in L_{0} .
\end{aligned}
$$

From $V_{0}$ and $V_{1}$, we have

$$
\begin{aligned}
V_{2}= & \mu^{-1}\left[i H_{0},\left[V_{1}, V_{0}\right]\right]=d_{11,21}^{(2)} y_{11,21}+d_{11,22}^{(2)} y_{11,22} \\
& +d_{21,31}^{(2)} y_{21,31}+d_{21,32}^{(2)} y_{21,32}+d_{22,31}^{(2)} y_{22,31}+d_{22,32}^{(2)} y_{22,32} \in L_{0},
\end{aligned}
$$

where the coefficients $d^{(2)}$ satisfy the following equations

$$
\begin{aligned}
& \left(\begin{array}{l}
d_{11,21}^{(2)} \\
d_{11,22}^{(2)}
\end{array}\right)=G_{1}\left(\begin{array}{l}
d_{11,21} \\
d_{11,22}
\end{array}\right), \quad G_{1}=\left(\begin{array}{cc}
v_{11,21} & -b_{1} \\
-b_{1} & v_{11,22}
\end{array}\right) \\
& \left(\begin{array}{l}
d_{21,31}^{(2)} \\
d_{21,32}^{(2)} \\
d_{22,31}^{(2)} \\
d_{22,32}^{(2)}
\end{array}\right)=G_{2}\left(\begin{array}{l}
d_{21,31} \\
d_{21,32} \\
d_{22,31} \\
d_{22,32}
\end{array}\right), G_{2}=\left(\begin{array}{cccc}
v_{21,31} & -b_{2} & b_{1} & 0 \\
-b_{2} & v_{21,32} & 0 & b_{1} \\
b_{1} & 0 & v_{22,31} & -b_{2} \\
0 & b_{1} & -b_{2} & v_{22,32}
\end{array}\right) .
\end{aligned}
$$

and the parameters $v_{i j, k l}$ and $b_{i}$ are defined as

$$
\begin{aligned}
& v_{11,21}=d_{21,31}^{2}+d_{21,32}^{2}-2 d_{11,21}^{2}-d_{11,22}^{2}, \\
& v_{11,22}=d_{22,31}^{2}+d_{22,32}^{2}-d_{11,21}^{2}-2 d_{11,22}^{2}, \\
& v_{21,31}=d_{11,21}^{2}-2 d_{21,31}^{2}-d_{21,32}^{2}-d_{22,31}^{2}, \\
& v_{21,32}=d_{11,21}^{2}-d_{21,31}^{2}-2 d_{21,32}^{2}-d_{22,32}^{2}, \\
& v_{22,31}=d_{11,22}^{2}-d_{21,31}^{2}-2 d_{22,31}^{2}-d_{22,32}^{2}, \\
& v_{22,32}=d_{11,22}^{2}-d_{21,32}^{2}-d_{22,31}^{2}-2 d_{22,32}^{2}, \\
& b_{1}=d_{11,21} d_{11,22}-d_{21,31} d_{22,31}-d_{21,32} d_{22,32}, \\
& b_{2}=d_{21,31} d_{21,32}+d_{22,31} d_{22,32} .
\end{aligned}
$$

As $G_{1}$ and $G_{2}$ are real symmetric matrices, we can diagonalize them by unitary transformation $U_{1}, U_{2}$, respectively

$$
\begin{aligned}
U_{1} G_{1} U_{1}^{-1} & =\left(\begin{array}{cc}
\lambda_{11} & 0 \\
0 & \lambda_{12}
\end{array}\right), \\
U_{2} G_{2} U_{2}^{-1} & =\left(\begin{array}{cccc}
\lambda_{21} & 0 & 0 & 0 \\
0 & \lambda_{22} & 0 & 0 \\
0 & 0 & \lambda_{23} & 0 \\
0 & 0 & 0 & \lambda_{24}
\end{array}\right),
\end{aligned}
$$

where $\lambda_{1 k}, \lambda_{2 p}$ are eigenvalues of $G_{1}$ and $G_{2}$, respectively. Introducing a set of new parameters

$$
\left(\begin{array}{l}
C_{11,21}^{(2)} \\
C_{11,22}^{(2)}
\end{array}\right)=U_{1}\left(\begin{array}{l}
d_{11,21}^{(2)} \\
d_{11,22}^{(2)}
\end{array}\right), \quad\left(\begin{array}{l}
C_{11,21} \\
C_{11,22}
\end{array}\right)=U_{1}\left(\begin{array}{l}
d_{11,21} \\
d_{11,22}
\end{array}\right),
$$


Complete controllability of finite quantum systems with two-fold energy level degeneracy10

$$
\left(\begin{array}{l}
C_{21,31}^{(2)} \\
C_{21,32}^{(2)} \\
C_{22,31}^{(2)} \\
C_{22,32}^{(2)}
\end{array}\right)=U_{2}\left(\begin{array}{l}
d_{21,31}^{(2)} \\
d_{21,32}^{(2)} \\
d_{22,31}^{(2)} \\
d_{22,32}^{(2)}
\end{array}\right), \quad\left(\begin{array}{l}
C_{21,31} \\
C_{21,32} \\
C_{22,31} \\
C_{22,32}
\end{array}\right)=U_{2}\left(\begin{array}{l}
d_{21,31} \\
d_{21,32} \\
d_{22,31} \\
d_{22,32}
\end{array}\right),
$$

we can easily obtain that

$$
\begin{array}{lll}
C_{11,21}^{(2)}=\lambda_{11} C_{11,21}, & C_{11,22}^{(2)}=\lambda_{12} C_{11,22}, & C_{21,31}^{(2)}=\lambda_{21} C_{21,31}, \\
C_{21,32}^{(2)}=\lambda_{22} C_{21,32}, & C_{22,31}^{(2)}=\lambda_{23} C_{22,31}, & C_{22,32}^{(2)}=\lambda_{24} C_{22,32} .
\end{array}
$$

In terms of those new parameters, $V_{1}$ can be rewritten as

$$
\begin{aligned}
& V_{1}=\left(y_{11,21}, y_{11,22}\right)\left(\begin{array}{l}
d_{11,21} \\
d_{11,22}
\end{array}\right)+\left(y_{21,31}, y_{21,32}, y_{22,31}, y_{22,32}\right)\left(\begin{array}{c}
d_{21,31} \\
d_{21,32} \\
d_{22,31} \\
d_{22,32}
\end{array}\right) \\
& =\left(y_{11,21}, y_{11,22}\right) U_{1}^{-1}\left(\begin{array}{l}
C_{11,21} \\
C_{11,22}
\end{array}\right)+\left(y_{21,31}, y_{21,32}, y_{22,31}, y_{22,32}\right) U_{2}^{-1}\left(\begin{array}{l}
C_{21,31} \\
C_{21,32} \\
C_{22,31} \\
C_{22,32}
\end{array}\right) \\
& =\left(\tilde{y}_{11,21}, \tilde{y}_{11,22}\right)\left(\begin{array}{l}
C_{11,21} \\
C_{11,22}
\end{array}\right)+\left(\tilde{y}_{21,31}, \tilde{y}_{21,32}, \tilde{y}_{22,31}, \tilde{y}_{22,32}\right)\left(\begin{array}{l}
C_{21,31} \\
C_{21,32} \\
C_{22,31} \\
C_{22,32}
\end{array}\right) \\
& =C_{11,21} \tilde{y}_{11,21}+C_{11,22} \tilde{y}_{11,22}+C_{21,31} \tilde{y}_{21,31}+C_{21,32} \tilde{y}_{21,32} \\
& +C_{22,31} \tilde{y}_{22,31}+C_{22,32} \tilde{y}_{22,32} \text {, }
\end{aligned}
$$

where

$$
\begin{aligned}
& \left(\tilde{y}_{11,21}, \tilde{y}_{11,22}\right)=\left(y_{11,21}, y_{11,22}\right) U_{1}^{-1}, \\
& \left(\tilde{y}_{21,31}, \tilde{y}_{21,31}, \tilde{y}_{21,31}, \tilde{y}_{21,31}\right)=\left(y_{21,31}, y_{21,31}, y_{21,31}, y_{21,31}\right) U_{2}^{-1} .
\end{aligned}
$$

Similarly, we have

$$
\begin{aligned}
V_{2}= & C_{11,21}^{(2)} \tilde{y}_{11,21}+C_{11,22}^{(2)} \tilde{y}_{11,22}+C_{21,31}^{(2)} \tilde{y}_{21,31} \\
& +C_{21,32}^{(2)} \tilde{y}_{21,32}+C_{22,31}^{(2)} \tilde{y}_{22,31}+C_{22,32}^{(2)} \tilde{y}_{22,32} \\
= & \lambda_{11} C_{11,21} \tilde{y}_{11,21}+\lambda_{12} C_{11,22} \tilde{y}_{11,22}+\lambda_{21} C_{21,31} \tilde{y}_{21,31} \\
& +\lambda_{22} C_{21,32} \tilde{y}_{21,32}+\lambda_{23} C_{22,31} \tilde{y}_{22,31}+\lambda_{24} C_{22,32} \tilde{y}_{22,32},
\end{aligned}
$$

Observing the Eqs.(56|59), both elements are linear combition of six elements $\tilde{y}_{i j, k l}$. In the following we always require that

$$
\lambda_{11} \neq 0, \quad \lambda_{12} \neq 0, \quad \lambda_{11} \neq \lambda_{12}, \quad C_{11,21} \neq 0, \quad C_{11,22} \neq 0 .
$$

If all other $\lambda_{2 k}(k=1,2,3,4)$ are vanishing, we can find that

$$
\tilde{y}_{11,21} \in L_{0}, \quad \tilde{y}_{11,22} \in L_{0} .
$$

If some $\lambda_{2 k} \neq 0$, we need more than Lie elements, which can be obtained by evaluating the following commutation relations $V_{m}=\mu^{-1}\left[i H_{0},\left[V_{m-1}, V_{0}\right]\right]$. It is easy to check that

$$
\begin{aligned}
V_{m}= & \lambda_{11}^{m-1} C_{11,21} \tilde{y}_{11,21}+\lambda_{12}^{m-1} C_{11,22} \tilde{y}_{11,22}+\lambda_{21}^{m-1} C_{21,31} \tilde{y}_{21,31} \\
& +\lambda_{22}^{m-1} C_{21,32} \tilde{y}_{21,32}+\lambda_{23}^{m-1} C_{22,31} \tilde{y}_{22,31}+\lambda_{24}^{m-1} C_{22,32} \tilde{y}_{22,32} \in L_{0} .
\end{aligned}
$$


Complete controllability of finite quantum systems with two-fold energy level degeneracy11

We then obtain $M$ those type of elements by choosing $m=1, \cdots, M$, where $M$ is the number of non-zero $\lambda_{i j, k l}$ 's. The coefficient matrix of those element is Vandermonde's matrix with non-zero determinant when all non-zero $\lambda$ 's are different from any others. If this condition is satisfied, we again obtain the Lie algebra element (61). As the transformation matrix $U_{1}$ is nonsingular, we obtain $y_{11,21}, y_{11,22} \in L_{0}$. In conclusion, the system with equal energy gaps is completely controllable if conditions (60) (18) are satisfied and all non-zero $\lambda_{i j}$ 's are distinctive.

We remark that if all $\lambda_{i j} \neq 0$ and $C_{i j, k l} \neq 0$, we can obtain all six elements $y_{i j, k l} \in L_{0}$ from six elements $V_{m}$ and then the generated Lie algebra is $\mathrm{SU}(5)$. In this case, we do not need to use Lemma 1 and thus the condition (18) is unnecessary.

\subsection{Controllability of systems with different energy gaps}

Now let us turn to the control of arbitrary $N$-dimensional quantum systems. We first consider in this subsection the system with distinct energy gaps.

Theorem 1 If $\mu_{1} \neq \mu_{n}, n=2,3, \ldots, N-1$, and the coupling constants satisfy the following conditions

$$
d_{11,21}\left(p d_{22,31}+q d_{22,32}\right) \neq d_{11,22}\left(p d_{21,31}+q d_{21,32}\right),
$$

where $p=d_{11,21} d_{21,31}+d_{11,22} d_{22,31}, q=d_{11,21} d_{21,32}+d_{11,22} d_{22,32}$, we have $y_{11,21}$, $y_{11,22} \in L_{0}$ and thus the system is completely controllable.

Proof. We evaluate

$$
\begin{aligned}
& V^{\prime}=\left[i H_{0}, i H_{1}\right]=\sum_{n=1}^{N-1} \sum_{j=1}^{\beta_{n}} \sum_{k=1}^{2} \mu_{n} d_{n j, n+1 k} y_{n j, n+1 k} \in L_{0}, \\
& V^{\prime \prime}=-\left[i H_{0}, V^{\prime}\right]=\sum_{n=1}^{N-1} \sum_{j=1}^{\beta_{n}} \sum_{k=1}^{2} \mu_{n}^{2} d_{n j, n+1 k} x_{n j, n+1 k} \in L_{0} .
\end{aligned}
$$

From $V^{\prime}$ and $V^{\prime \prime}$, we have

$$
\begin{aligned}
V_{1} & =V^{\prime \prime}-\mu_{N-1}^{2}\left(i H_{1}\right) \\
& =\sum_{n=1}^{N-2} \sum_{j=1}^{\beta_{n}} \sum_{k=1}^{2}\left(\mu_{n}^{2}-\mu_{N-1}^{2}\right) d_{n j, n+1 k} x_{n j, n+1 k} \in L_{0} .
\end{aligned}
$$

Repeating this process for $i H_{0}$ and $V_{1}$ leads to

$$
\begin{aligned}
V_{2} & =-\left[i H_{0},\left[i H_{0}, V_{1}\right]\right]-\mu_{N-2}^{2} V_{1}, \\
& =\sum_{n=1}^{N-3} \sum_{j=1}^{\beta_{n}} \sum_{k=1}^{2}\left(\mu_{n}^{2}-\mu_{N-2}^{2}\right)\left(\mu_{n}^{2}-\mu_{N-1}^{2}\right) d_{n j, n+1 k} x_{n j, n+1 k} \in L_{0} .
\end{aligned}
$$

After $N-2$ iterations, we have

$$
V_{N-2}=\left[\prod_{n=1}^{N-1}\left(\mu_{1}^{2}-\mu_{n}^{2}\right)\right]\left(d_{11,21} x_{11,21}+d_{11,22} x_{11,22}\right) \in L_{0} .
$$

Since $\mu_{1}$ is different from any others, $\prod_{n=1}^{N-1}\left(\mu_{1}^{2}-\mu_{n}^{2}\right) \neq 0$. This means that

$$
V_{N-2}^{\prime}=d_{11,21} x_{11,21}+d_{11,22} x_{11,22} \in L_{0}
$$


Complete controllability of finite quantum systems with two-fold energy level degeneracy 12

and

$$
V_{N-2}^{\prime \prime}=\mu_{1}^{-1}\left[i H_{0}, V_{N-2}^{\prime}\right]=d_{11,21} y_{11,21}+d_{11,22} y_{11,22} \in L_{0} .
$$

To prove the complete controllability, we need to separate the Eq. (68) to get $x_{11,21}, x_{11,21} \in L_{0}$. Let us discuss this in two different cases.

Case 1: $N=3$. In this case, the commutator between $V_{N-2}^{\prime \prime}$ and $V_{0}$ is

$$
\begin{aligned}
X & =\left[\left[V_{N-2}^{\prime \prime}, V_{0}\right], V_{0}\right] \\
& =\left(p d_{21,31}+q d_{21,32}\right) x_{11,21}+\left(p d_{22,31}+q d_{22,32}\right) x_{11,22} .
\end{aligned}
$$

where $V_{0}$ is given in Eq. (20). From (68) and (70), we know that, if condition (63) is satisfied, $x_{11,21}, x_{11,21} \in L_{0}$. Then from Lemma 1, the system is completely controllable.

Case 2: $N>3$. In this case, the commutator

$$
\begin{aligned}
X= & {\left[\left[V_{N-2}^{\prime \prime}, V_{0}\right], V_{0}\right] } \\
= & \left(p d_{21,31}+q d_{21,32}\right) x_{11,21}+\left(p d_{22,31}+q d_{22,32}\right) x_{11,22}+ \\
& \left(p d_{31,41}+q d_{32,41}\right) x_{11,41}+\left(p d_{31,42}+q d_{32,42}\right) x_{11,42} \in L_{0},
\end{aligned}
$$

has two additional terms $x_{11,41}$ and $x_{11,42}$. Fortunately, we can remove those terms by evaluating

$$
\begin{aligned}
X^{\prime} & =\left[\mu_{1}^{2}-\left(\mu_{1}+\mu_{2}+\mu_{3}\right)^{2}\right]^{-1}\left(\left[i H_{0},\left[i H_{0}, X\right]\right]-\left(\mu_{1}+\mu_{2}+\mu_{3}\right)^{2} X\right) \\
& =\left(p d_{21,31}+q d_{21,32}\right) x_{11,21}+\left(p d_{22,31}+q d_{22,32}\right) x_{11,22} \in L_{0} .
\end{aligned}
$$

From Eqs. (6872), and the condition (63), we obtain $x_{11,21}, x_{11,22} \in L_{0}$, and moreover, $y_{11,21}, y_{11,22} \in L_{0}$. The conclusion then follows from Lemma 1 .

\subsection{Complete controllability of systems with equal energy gaps}

In this subsection we consider the system with equally spaced energy gaps, namely $\mu \equiv \mu_{1}=\mu_{2}=\cdots=\mu_{N-1} \neq 0$. Let us first introduce some parameters

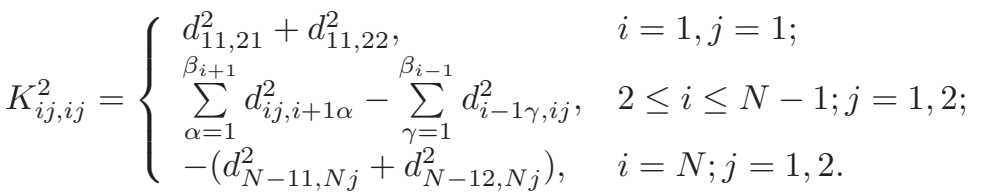

$$
\begin{aligned}
& \nu_{i j, i+1 k}=K_{i+1 K, i+1 K}^{2}-K_{i j, i j}^{2} \text {, }
\end{aligned}
$$

and

$$
\begin{aligned}
& b_{1}=d_{11,21} d_{11,22}-d_{21,31} d_{22,31}-d_{21,32} d_{22,32}, \\
& b_{i}=d_{i 1, i+11} d_{i 1, i+12}+d_{i 2, i+11} d_{i 2, i+12}-d_{i+11, i+21} d_{i+12, i+21} \\
& \quad-d_{i+11, i+22} d_{i+12, i+22}, \quad 2 \leq i \leq N-2 ; \\
& b_{N-1}=d_{N-11, N 1} d_{N-11, N 2}+d_{N-12, N 1} d_{N-12, N 2} .
\end{aligned}
$$

Let $V=i H_{1}$. We first observe that

$$
\widetilde{V}=\mu^{-1}\left[i H_{0}, i H_{1}\right]=\sum_{i=1}^{N-1} \sum_{j=1}^{\beta_{i}} \sum_{k=1}^{2} d_{i j, i+1 k} y_{i j, i+1 k} \in L_{0} .
$$


Complete controllability of finite quantum systems with two-fold energy level degeneracy 13

Sum and difference of $\widetilde{V}$ with $V$ give rise to

$$
\begin{aligned}
& V_{1}^{+}=\sum_{i=1}^{N-1} \sum_{j=1}^{\beta_{i}} \sum_{k=1}^{2} d_{i j, i+1 k}\left(x_{i j, i+1 k}+y_{i j, i+1 k}\right), \\
& V_{1}^{-}=\sum_{i=1}^{N-1} \sum_{j=1}^{\beta_{i}} \sum_{k=1}^{2} d_{i j, i+1 k}\left(x_{i j, i+1 k}-y_{i j, i+1 k}\right),
\end{aligned}
$$

which, along with their commutator

$$
V_{1}^{0}=\frac{1}{4}\left[V_{1}^{+}, V_{1}^{-}\right]=i \sum_{i=1}^{N} \sum_{j=1}^{\beta_{i}} K_{i j, i j}^{2} e_{i j, i j}-\sum_{i=1}^{N-1} b_{i} x_{i+11, i+22},
$$

are all in $L_{0}$. Starting from $\widetilde{V}$ and $V_{1}^{0}$, we have

$$
\widetilde{V}_{1}=\mu^{-1}\left[i H_{0},\left[\widetilde{V}, V_{1}^{0}\right]\right]=\sum_{i=1}^{N-1} \sum_{j=1}^{\beta_{i}} \sum_{k=1}^{2} d_{i j, i+1 k}^{(2)} y_{i j, i+1 k},
$$

where we have assumed $b_{0}=0$ and introduced the notation

$$
d_{i j, i+1 k}^{(2)} \equiv \nu_{i j, i+1 k} d_{i j, i+1 k}-b_{i} d_{i j, i+1 p}+b_{i-1} d_{i \alpha, i+1 k}, p \neq k, \alpha \neq j .
$$

Repeating the process for $n$ times, we obtain the element $\widetilde{V}_{n}$

$$
\widetilde{V}_{n}=\sum_{i=1}^{N-1} \sum_{j=1}^{\beta_{i}} \sum_{k=1}^{2} d_{i j, i+1 k}^{(n+1)} y_{i j, i+1 k}
$$

where the coefficients satisfy Eq.(48) for $i=1$ and the following recursion relations

$$
\left(\begin{array}{l}
d_{i, 1+11}^{(n+1)} \\
d_{i 1, i+12}^{(n+1)} \\
d_{i 2, i+11}^{(n+1)} \\
d_{i 2, i+12}^{(n+1)}
\end{array}\right)=G_{i}\left(\begin{array}{c}
d_{i, i+11}^{(n)} \\
d_{i 1, i+12}^{(n)} \\
d_{i 2, i+11}^{(n)} \\
d_{i 2, i+12}^{(n)}
\end{array}\right),
$$

where

$$
G_{i}=\left(\begin{array}{cccc}
\nu_{i 1, i+11} & -b_{i} & b_{i-1} & 0 \\
-b_{i} & \nu_{i 1, i+12} & 0 & b_{i-1} \\
b_{i-1} & 0 & \nu_{i 2, i+11} & -b_{i} \\
0 & b_{i-1} & -b_{i} & \nu_{i 2, i+12}
\end{array}\right)
$$

is independent of $n$. Noting that the coefficient matrix $G_{1}$ given in (48) and $G_{i}$ is a real symmetric matrix and can be diagonalized through unitary transformations $U_{1}$ (see Eq. (510) and $U_{i}$

$$
U_{i} G_{i} U_{i}^{-1}=\left(\begin{array}{cccc}
\lambda_{i 1} & 0 & 0 & 0 \\
0 & \lambda_{i 2} & 0 & 0 \\
0 & 0 & \lambda_{i 3} & 0 \\
0 & 0 & 0 & \lambda_{i 4}
\end{array}\right)
$$

where the diagonal elements $\lambda_{i j}(j=1,2,3,4)$ are the eigenvalue of $G_{i}$.

Introduce a set of new parameters

$$
\left(\begin{array}{c}
C_{i 1, i+11}^{(n+1)} \\
C_{i 1, i+12}^{(n+1)} \\
C_{i 2, i+11}^{(n+1)} \\
C_{i 2, i+12}^{(n+1)}
\end{array}\right)=G_{i}\left(\begin{array}{c}
d_{i 1, i+11}^{(n+1)} \\
d_{i 1, i+12}^{(n+1)} \\
d_{i 2, i+11}^{(n+1)} \\
d_{i 2, i+12}^{(n+1)}
\end{array}\right) .
$$


Complete controllability of finite quantum systems with two-fold energy level degeneracy 14

Then we can easily obtain the recurrence relations between these new parameters

$$
\begin{array}{ll}
C_{i 1, i+11}^{(n+1)}=\lambda_{i 1} C_{i 1, i+11}^{(n)}, & C_{i 1, i+12}^{(n+1)}=\lambda_{i 2} C_{i 1, i+12}^{(n)}, \\
C_{i 2, i+11}^{(n+1)}=\lambda_{i 3} C_{i 2, i+11}^{(n)}, & C_{i 2, i+12}^{(n+1)}=\lambda_{i 4} C_{i 2, i+12}^{(n)} .
\end{array}
$$

We can rewritten the element (81) as

$$
\widetilde{V}_{n}=\sum_{i=1}^{N-1} \sum_{j=1}^{\beta_{i}} \sum_{k=1}^{2} C_{i j, i+1 k}^{(n+1)} \tilde{y}_{i j, i+1 k}=\sum_{i=1}^{N-1} \sum_{j=1}^{\beta_{i}} \sum_{k=1}^{2} \lambda_{i p_{j k}}^{n} C_{i j, i+1 k}^{(1)} \tilde{y}_{i j, i+1 k} \in L_{0},
$$

where $m=1,2, \ldots, M, p_{11}=1, p_{12}=2, p_{21}=3, p_{22}=4$, and

$$
\left(\tilde{y}_{i 1, i+11}, \tilde{y}_{i 1, i+12}, \tilde{y}_{i 2, i+11}, \tilde{y}_{i 2, i+12}\right)=\left(y_{i 1, i+11}, y_{i 1, i+12}, y_{i 2, i+11}, y_{i 2, i+12}\right) U_{i}^{-1} \text {. }
$$

Let $M$ be the number of non-zero $C_{i j, i+1 k}^{(1)} \tilde{y}_{i j, i+1 k}$ in (87). Then we get a set of equations about $C_{i j, i+1 k}^{(1)} \tilde{y}_{i j, i+1 k}$ whose coefficient matrix is the square Vandermonde's matrix. If the determinant of coefficient matrix is not vanishing, or in other words, all $\lambda_{i j}$ are different from any others, we can obtain $C_{11,21}^{(1)} \tilde{y}_{11,21}, C_{11,22}^{(1)} \tilde{y}_{11,22} \in L_{0}$ and further $y_{11,21}, y_{11,22} \in L_{0}$ due to $C_{11,21}^{(1)}, C_{11,22}^{(1)} \neq 0$ and the unitarity of the matrix $U_{i}$. From Lemma 1 we conclude that $L_{0}=\mathrm{su}(2 N-1)$ and the system is completely controllable.

In summary, we conclude that

Theorem 2 The degenerate quantum system with $N$ equally spaced energy levels is completely controllable if the parameters satisfy the following conditions

- Condition (18) for the validity of Lemma 1;

- Condition (60);

- All non-zero $\lambda_{i j}$ are different from any others to guarantee determinant of Vandermonde's matrix non-vanishing.

As an explicit example, one can check that the degenerate system with $E_{n}=$ $n-1 / 2$ and $d_{i j, i+1 k}=(N+3-i-j-k)^{1 / 2}$ is completely controllable.

\section{Conclusion}

In this paper, we have systematically investigated the control of quantum system with energy degeneracy using two different approach. The first approach is to apply a weak constant field to eliminate the degeneracy and then control it using techniques developed for non-degenerate quantum system. We first examine the conditions for the elimination of degeneracy and then address the issue of influence of relaxation time of constant external field to the target state by calculating the fidelity.

We then investigate the completely controllability of degenerate system by a single control field only. It is found that the two level system is not completely controllable in this control scheme. But fortunately, the multi-level system with more than two energy levels are completely controllable if the energy gap and the value of the transition dipole moments $d_{n k, n+1 p}$ satisfy some conditions. Two different cases, namely the system with different energy gaps and with equal energy gaps, are considered as in the non-degenerate case.

In the forthcoming papers we shall consider the control the quantum system with general degeneracy degree rather than just 2 in this paper. Such investigation might find applications in the control of molecular system and chain of qubits in quantum computation. 
Complete controllability of finite quantum systems with two-fold energy level degeneracy 15

\section{Acknowledgement}

This work is supported in part by NFRPC 973 Project under grand number 2006CB921205.

[1] 1987 Information Complexity and Control in Quantum Physics ed A Blaquiere, S Dinerand and G Lochak (New York: Springer)

[2] Butkovskiy A G and Samoilenko Yu I 1990 Control of Quantum-Mechanical Processes and Systems (Dordrecht: Kluwer)

[3] Jurdjevic V 1997 Geometric Control Theory (Cambridge: Cambridge University Press)

[4] Lloyd S 2000 Phys. Rev. A 62022108

[5] Huang G M, Tarn T J and Clark J W 1983 J. Math. Phys. 242608

[6] Ramakrishna V and Rabitz H 1996 Phys. Rev. A 541715

[7] Rabitz H, de Vivie-Riedle R, Motzkus M and Kompa K 2000 Science 288824

[8] Schirmer S G, Solomon A I and Leahy J V 2002 J. Phys. A 354125

[9] Fu H, Schirmer S G and Solomon A I 2001 J. Phys. A: Math. Gen. 341679

[10] Schirmer S G, Fu H and Solomon A I 2001 Phys. Rev. A 63063410

[11] Turinici G 2000 Mathematical Models and Methods for ab Initio Quantum Chemistry (Lecture Notes in Chemistry vol 74) ed M Defranceschi and C Le Bris (Berlin: Springer)

[12] Turinici G and Rabitz H 2001 Chem. Phys. 2671

[13] Rangan C and Bloch A M 2005 J. Math. Phys. 46032106

[14] Cabrera R, Bayis W E, Rangan C 2007 Phys. Rev. A. 76033401 Albertini F and D'Alessandro D 2003 IEEE Trans. Autom. Control 481399

[15] Brockett R W 1973 SIAM J. Appl. Math. 25213 Brockett R W 1973 Lie Algebras and Lie Groups in Control Theory Geometric Methods in System Theory ed D Q Mayne and R W Brockett (Dordrecht: Reidel) pp 43C82

[16] Fu H C, Dong H, Liu X F and Sun C P 2007 Phys. Rev. A 75052317 Fu H C, Dong H, Liu X F and Sun C P 2009 J. Phys. A: Math. Theor. 42045303

[17] Humphreys J E 1972 Introduction to Lie Algebras and Representation Theory (New York: Springer)

[18] Landau L D and Lifshitz E M 1977 Quantum Mechanics (Non-relativistic Theory) (Reed Educational and Professional Publishing Ltd, Third Edition) 